\title{
Elastic Purcell Effect
}

\author{
Mikołaj K. Schmidt, ${ }^{1,2, *}$ L. G. Helt, ${ }^{3}$ Christopher G. Poulton, ${ }^{1,4}$ and M. J. Steel ${ }^{1,2}$ \\ ${ }^{1}$ Centre for Ultrahigh bandwidth Devices for Optical Systems (CUDOS), Australia \\ ${ }^{2}$ Macquarie University Research Centre in Quantum Science and Technology (QSciTech), \\ MQ Photonics Research Centre, Department of Physics and Astronomy, Macquarie University, \\ New South Wales 2109, Australia \\ ${ }^{3}$ Department of Physics, Engineering Physics \& Astronomy, Queen's University, \\ Kingston, Ontario, K7L 3N6, Canada \\ ${ }^{4}$ School of Mathematical and Physical Sciences, University of Technology Sydney, \\ New South Wales 2007, Australia
}

(Received 14 January 2018; published 8 August 2018)

\begin{abstract}
In this work, we introduce an elastic analog of the Purcell effect and show theoretically that spherical nanoparticles can serve as tunable and robust antennas for modifying the emission from localized elastic sources. This effect can be qualitatively described by introducing elastic counterparts of the familiar electromagnetic parameters: local density of elastic states, elastic Purcell factor, and effective volume of elastic modes. To illustrate our framework, we consider the example of a submicron gold sphere as a generic elastic $\mathrm{GHz}$ antenna and find that shear and mixed modes of low orders in such systems offer considerable elastic Purcell factors. This formalism opens pathways towards extended control over dissipation of vibrations in various optomechanical systems and contributes to closing the gap between classical and quantum-mechanical treatments of phonons localized in elastic nanoresonators.
\end{abstract}

DOI: 10.1103/PhysRevLett.121.064301

In 1946 [1], E. M. Purcell proposed that the rate of nuclear magnetic moment transitions can be increased by coupling the system to an electric circuit. This enhancement, the "Purcell effect," depends on the fundamental properties of the circuit or, more generally, the mode to which the system is coupled: its resonant frequency, volume $(V)$, and quality factor $(Q)$. Ever since, considerable effort has been devoted to designing tunable resonators with ever smaller mode volumes and larger $Q$ s. Recently, a similar modification of the decay rate has been demonstrated in the acoustic domain by measuring changes in the damping of oscillations of a Chinese gong placed near a hard wall [2]. While that system does not exhibit any resonant behavior, or a clear cavity-like structure, it suggests that the original idea from Purcell can be extended to the physics of mechanical vibrations. In enabling such a direct analog, we provide a robust framework for calculating, and ultimately engineering, the output of an elastic source. The ability to control the rate of mechanical emission can be extended to spatial and spectral degrees of freedom by engineering the local density of elastic states (LDES) with resonant elastic nanosystems and leads to the possibility of manipulating phonon-mediated nonlinear optical processes [3-6].

In this Letter, we investigate the elastic Purcell factor by analyzing the response of elastic antennas formed by spherical nanoparticles and discuss how they can enhance or quench the emission from nearby sources of elastic waves. To go beyond purely acoustic phenomena [2,7], we adopt the theory of elastic wave scattering [8-12] which accounts for the presence of both longitudinal and transverse waves propagating in the elastic medium. As an illustration, we calculate the elastic scattering cross sections of a generic optical nanoantenna-a gold spherical nanoparticle-and discuss in detail the resonances found in such a system. We show how these resonators act as efficient and selective elastic antennas for both longitudinal and transverse radiation from localized elastic emitters, substantially suppressing or enhancing their emission rates. The elastic modes of the resonator are further characterized by introducing physical parameters analogous to those used in electromagnetism, such as an elastic Purcell factor, or an effective mode volume. While we limit our investigations in this Letter to a harmonic point force coupled to the displacement field, we plan to extend our formalism to additional sources for consideration of complex systems in future work. Similar modifications of the optical Purcell effect have been introduced to describe the coupling of magnetic dipolar, or multipolar emitters, to electromagnetic waves [13]. This framework offers a direct path towards a quantum-mechanical description of the interaction in elastic nanosystems and can be readily applied to explore other phenomena in the weak- and strong-elastic-coupling regimes.

The generic elastic nanoantenna shown in Fig. 1(a) is a homogeneous isotropic solid sphere characterized by Lamé parameters $\lambda_{2}, \mu_{2}$, and density $\rho_{2}$ and the velocities of longitudinal and transverse waves [14]. We consider the 
(a)

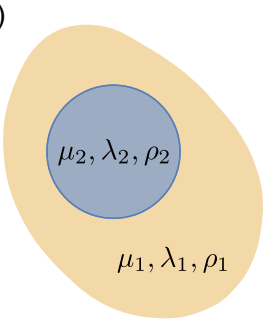

(b)

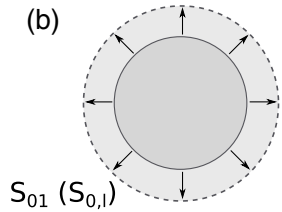

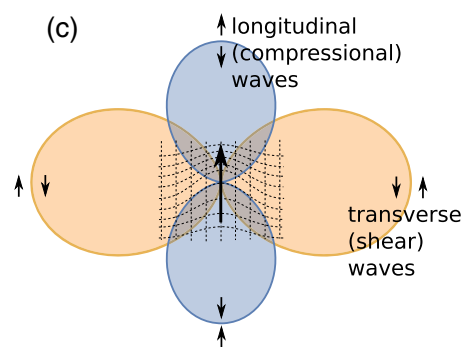

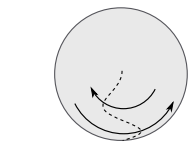

$T_{11}\left(T_{n, l-1}\right)$

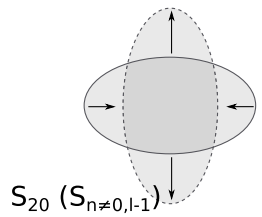

FIG. 1. Schematics of (a) an elastic spherical nanoantenna, (b) its three families of modes, and (c) elastic localized emitters. (a) The antenna (medium) is characterized by its density $\rho_{2}\left(\rho_{1}\right)$ and two Lamé parameters: $\lambda_{2}\left(\lambda_{1}\right)$ and $\mu_{2}\left(\mu_{1}\right)$. (b) Elastic modes of spherical resonators (left to right): compressional $S_{0, l}$, pure shear $T_{n, l-1}$, and mixed $S_{n \neq 0, l-1}$. (c) Radiation pattern from an elastic emitter (large black arrow) in an isotropic, homogeneous elastic medium. Transverse (shear) waves carry the energy in the plane perpendicular to the axis of the emitter, while the longitudinal (compressional) waves radiate along it. Small black arrows depict the orientation of displacement fields in the far field, and the dashed grid illustrates the displacement field in the near field.

surrounding medium to be similarly isotropic, solid, and homogeneous, with parameters $\lambda_{1}, \mu_{1}, \rho_{1}$, and supporting both transverse and longitudinal waves. To pursue elastic systems resonant at the typical mechanical frequencies of interest - a few GHz [15-17]-we can consider submicron metallic spheres [18-20]. Coincidentaly, such particles exhibit large optical Purcell factors and have therefore been explored as building blocks for optical nanodevices $[13,21,22]$. Rescaling towards $\mathrm{THz}$ frequencies characteristic of Raman scattering [23] would likely be limited by the large viscosity, and hence elastic loss of the composite materials in this regime.

To establish the formalism used throughout this work, let us consider the decomposition of the displacement field $\mathbf{u}$ into longitudinal $\left(\mathbf{u}_{l}\right)$ and shear $\left(\mathbf{u}_{s}\right)$ monochromatic waves propagating in the environment with Lamé parameters $\lambda_{1}$ and $\mu_{1}$ at velocities $v_{l, 1}$ and $v_{s, 1}$, respectively [8-12]. In the homogeneous isotropic medium, these displacement fields satisfy the wave equation $\omega^{2} \chi+v_{\chi}^{2} \nabla^{2} \chi=0$ for $\left(\chi, v_{\chi}\right)=$ $\left(\mathbf{u}_{s}, v_{s, 1}\right)$ and $\left(\chi, v_{\chi}\right)=\left(\mathbf{u}_{l}, v_{l, 1}\right)$. We expand the shear field into two families of vector spherical harmonics (VSHs):

$$
\mathbf{M}_{m n}=\nabla \times\left(\mathbf{r} \Psi_{m n}\right), \quad \mathbf{N}_{m n}=\frac{v_{s}}{\omega} \nabla \times \mathbf{M}_{m n}
$$

defined by a scalar potential $\Psi_{m n}$ fulfilling the scalar wave equation with velocity $v_{s, 1}\left[\left(\chi, v_{\chi}\right)=\left(\Psi_{m n}, v_{s, 1}\right)\right]$. As for EM, the transverse VSHs are constructed from the

associated Legendre polynomials $P_{n}^{m}$ and spherical Bessel functions $j_{n}, y_{n}, h_{n}^{(1)}$, and $h_{n}^{(2)}$ [24]. Similarly, the longitudinal waves propagating in the medium are described by one family of VSHs $\mathbf{L}_{m n}=\nabla \Phi_{m n}$ derived from the scalar solution $\Phi_{m n}$ to the wave equation with velocity $v_{l, 1}$. Full expressions for all the VSHs can be found in Appendix A in the Supplemental Material [25] and in Refs. [8,9].

These three families of VSHs can be used to represent any physical wave impinging on the scattering sphere and can simultaneously be used to characterize the quasimodes of the vibrating sphere, shown in Fig. 1(b). Compressional modes, denoted usually as $S_{0, l}[14,26]$, which include the lowest-order breathing mode [Fig. 1(b), left panel], are given by the $\mathbf{L}_{m n}$ VSHs; pure shear (or torsional) modes $T_{n, l-1}$ with vanishing radial displacement fields are given by the $\mathbf{M}_{m n}$ VSHs and include the lowest-order $T_{11}$ mode depicted in the middle panel in Fig. 1(b). Finally, mixed modes $S_{n \neq 0, l-1}$ include the lower-order mode shown in the right panel in Fig. 1(b), in which compression along one axis induces expansion in the normal plane. The modes of this last family are described by linear combinations of the $\mathbf{N}_{m n}$ and $\mathbf{L}_{m n}$ harmonics. Note that while subscript $n$ corresponds to the radial dependence of both the modes of the sphere and the VSHs, subscript $l$ indexes the resonant frequencies for a given $n$ and is, in general, not related to the azimuthal number $m$ of the VSH [14].

Since we identify three families of elastic VSHs, in contrast to two found in electromagnetic Mie theory, we can expect that the elastic boundary conditions (BCs) between the medium and the sphere will be more involved. Indeed, they require that all components of the displacement field $\mathbf{u}$, as well as normal components of the stress field $\mathbf{T} \cdot \hat{\mathbf{n}}$ be continuous. For the sphere centered at the origin of the coordinate system, these normal components correspond to the radial elements of the stress tensor $\mathbf{T} \cdot \hat{\mathbf{r}}=\left(T_{r r}, T_{r \theta}, T_{r \phi}\right)$ :

$$
\begin{gathered}
\frac{T_{r r}}{2 \rho v_{s}^{2}}=\frac{\sigma \nabla \cdot \mathbf{u}}{1-2 \sigma}+u_{r, r}, \quad \frac{T_{r \theta}}{\mu}=u_{\theta, r}-\frac{u_{\theta}}{r}+\frac{u_{r, \theta}}{r}, \\
\frac{T_{r \phi}}{\mu}=\frac{u_{r, \phi}}{r \sin (\theta)}+u_{\phi, r}-\frac{u_{\phi}}{r}
\end{gathered}
$$

where $u_{i, j}=\partial_{j} u_{i}$, and Poisson's ratio $\sigma$ is related to the Lamé parameters through $\sigma=\lambda /[2(\lambda+\mu)]$ [14]. By numerically solving these BCs in the truncated basis of VSHs (see Appendix B in the Supplemental Material [25] and Refs. [8,9]), we identify eigenmodes of the system characterized by complex eigenfrequencies. Following the literature on cavity QED, we dub these quasinormal modes (QNMs) [21]. The QNMs identified in the nanoantenna system include pure shear, mixed, and compressional modes described earlier.

In Fig. 2, we present a comparison of the scattering efficiency (defined as the scattering cross section 


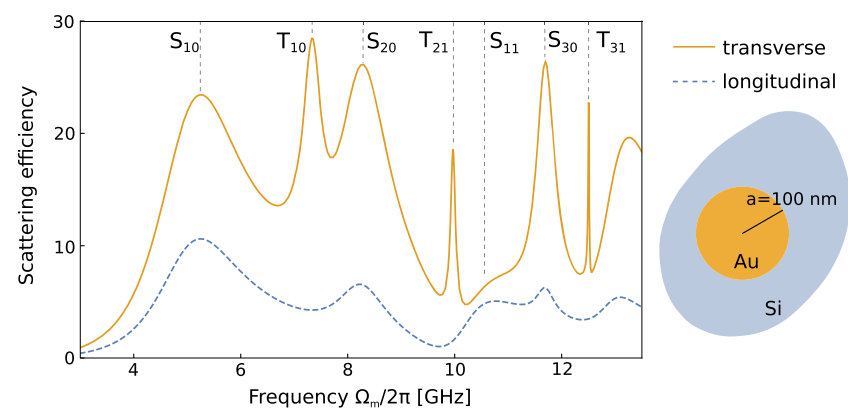

FIG. 2. Scattering efficiencies of elastic longitudinal (blue dashed lines) and transverse (orange solid lines) plane waves by a spherical submicron radius gold resonator immersed in silicon in the lowest-order resonance region [27].

normalized by the geometric cross section of the sphere; see Appendix B3 in the Supplemental Material [25]) of transverse and longitudinal plane wave excitations for a spherical $100 \mathrm{~nm}$ radius gold nanoantenna $\left(\lambda_{\mathrm{Au}}=154 \mathrm{GPa}\right.$, $\mu_{\mathrm{Au}}=30 \mathrm{GPa}, \rho_{\mathrm{Au}}=19.3 \mathrm{~g} / \mathrm{cm}^{3}$ ) immersed in silicon $\left(\lambda_{\mathrm{Si}}=52 \mathrm{GPa}, \mu_{\mathrm{Si}}=66 \mathrm{GPa}, \rho_{\mathrm{Si}}=2.3 \mathrm{~g} / \mathrm{cm}^{3}\right)$. The lowest energy mixed $\left(S_{10}, S_{20}, S_{11}, S_{30}\right)$ and pure shear modes $\left(T_{10}, T_{21}, T_{31}\right)$ are marked with dashed lines. Shear QNMs, resonant at $\omega_{T_{10}} / 2 \pi \approx 7.34 \mathrm{GHz}, \omega_{T_{21}} / 2 \pi \approx 9.97 \mathrm{GHz}$, $\omega_{T_{31}} / 2 \pi \approx 12.5 \mathrm{GHz}$, are characterized by quality factors from 18 to 550. Since these modes are accessible only to transverse plane waves, they would not be easily excited if the medium supported solely longitudinal, pressurelike waves. Compressional modes are found for larger frequencies, with $\omega_{S_{01}} / 2 \pi \approx 23.9 \mathrm{GHz}$ and $Q_{S_{01}} \approx 11$.

Having analyzed the response of generic elastic nanoantennas, we can consider their application for controlling the dynamics of nearby phononic emitters. A number of such elastic sources have been recently reported, exhibiting both bosonic [28,29] and fermionic $[16,17,30]$ statistics of the emitted phonons, and are being investigated for applications in phonon-based quantum networks [15].

The choice of model for an elastic emitter, and its coupling to the mechanical vibration of the environment, depends on the particular physical realization of the source. In this work, we consider the simplest scenario and model the elastic emitter as an oscillating, localized linear force $\mathbf{f} e^{-i \Omega t}$. This form of an elastic source can be realized in various optomechanical systems, e.g., by considering magnetostrictive inclusions in the structure [31] or an absorbing nanoparticle vibrating due to harmonic radiation pressure [32]. We note that such a canonical optomechanical setup has been realized in numerous studies [33] and that the elastic radiation rate has proven to be the critical factor in reaching interesting regimes of cooling of the mechanical degree of freedom as well as phonon lasing.

For such an emitter embedded in a homogeneous, lossless medium, we calculate the power of the emitted elastic radiation from the work done by the force on the displacement field $\mathbf{u}\left(\mathbf{r}_{m}\right) e^{-i \Omega t}$ at the position of the emitter $\left(\mathbf{r}_{m}\right)[14,34]:$

$$
P_{0}=\frac{d W}{d t}=\frac{\Omega_{m}}{2} \operatorname{Im}\left[\mathbf{u}\left(\mathbf{r}_{m}\right) \cdot \mathbf{f}^{*}\right] .
$$

This radiation will dissipate the energy of the emitter with rate $\gamma_{0}$ [34]. The displacement $\mathbf{u}\left(\mathbf{r}_{m}\right)$ can be found by applying the definition of the elastic Green function $\mathbf{u}(\mathbf{r})=\mathbf{G}\left(\mathbf{r}, \mathbf{r}_{m}\right) \mathbf{f}\left(\mathbf{r}_{m}\right)$. In the environment characterized by $\left(\lambda_{1}, \mu_{1}, \rho_{1}\right)$, in terms of $\mathbf{d}=\mathbf{r}-\mathbf{r}_{m}$, the dyadic Green function is $[35,36]$

$$
\begin{aligned}
\mathbf{G}_{0}(\mathbf{d})= & \frac{i k_{l 1}}{12 \pi\left(\lambda_{1}+2 \mu_{1}\right)}\left[\mathbb{1} h_{0}^{(1)}\left(k_{l 1} d\right)+\left(\mathbb{1}-3 \hat{\mathbf{d}} \hat{\mathbf{d}}^{T}\right) h_{2}^{(1)}\left(k_{l 1} d\right)\right] \\
& -\frac{i k_{s 1}}{12 \pi \mu_{1}}\left[-2 \mathbb{1} h_{0}^{(1)}\left(k_{s 1} d\right)+\left(\mathbb{1}-3 \hat{\mathbf{d}} \hat{\mathbf{d}}^{T}\right) h_{2}^{(1)}\left(k_{s 1} d\right)\right],
\end{aligned}
$$

where $\mathbb{1}$ is the identity tensor, $h_{0}^{(1)}$ and $h_{2}^{(1)}$ are the spherical Hankel functions of the first kind of the 0th and 2nd order. Here, $k_{l 1}=\Omega / v_{l 1}$ and $k_{s 1}=\Omega / v_{s 1}$ are the wave numbers associated with longitudinal and transverse (shear) waves, respectively.

The second part of the Green function, describing the shear waves, has a form similar to that of the electromagnetic Green function, ensuring that the shear waves propagate in a dipole-like pattern, with the largest emission in the plane perpendicular to the axis of the force $(\theta=0)$. The longitudinal waves have an identical near- and intermediate-field distribution (except for the wave number), but the far field has a $\cos ^{2} \theta$-like distribution [see Fig. 1(c) and Appendix A in the Supplemental Material [25]].

The radiated power $P_{0}$ in the homogeneous medium can be calculated from the Green function (see Appendix $\mathrm{C}$ in the Supplemental Material [25]) as

$$
P_{0}=\frac{\Omega|\mathbf{f}|^{2}}{12 \pi}\left(\frac{k_{s 1}}{\mu}+\frac{k_{l 1}}{2\left(\lambda_{1}+2 \mu_{1}\right)}\right) .
$$

Unlike for the power emitted by an electromagnetic dipolar emitter, the elastic $P_{0}$ exhibits quadratic, rather than quartic, dependence on the emission frequency $\Omega$. This is due to the difference in the dispersion of the inhomogeneous term in the wave equations for electric field $\mathbf{E}$ compared to displacement $\mathbf{u}$ [14].

The radiation of an emitter in an arbitrary system can be calculated by expanding Eq. (4), including the Green function that represents scattering on an elastic antenna $\mathbf{G}=\mathbf{G}_{0}+\mathbf{G}_{S}$, and writing the enhancement as

$$
\frac{P}{P_{0}}=1+\frac{6 \pi}{|\mathbf{f}|^{2}} \frac{\operatorname{Im}\left\{\mathbf{f}^{*} \cdot \mathbf{G}_{S}\left(\mathbf{r}_{m}, \mathbf{r}_{m}\right) \cdot \mathbf{f}\right\}}{\frac{k_{l 1}}{2\left(\lambda_{1}+2 \mu_{1}\right)}+\frac{k_{s 1}}{\mu_{1}}} .
$$




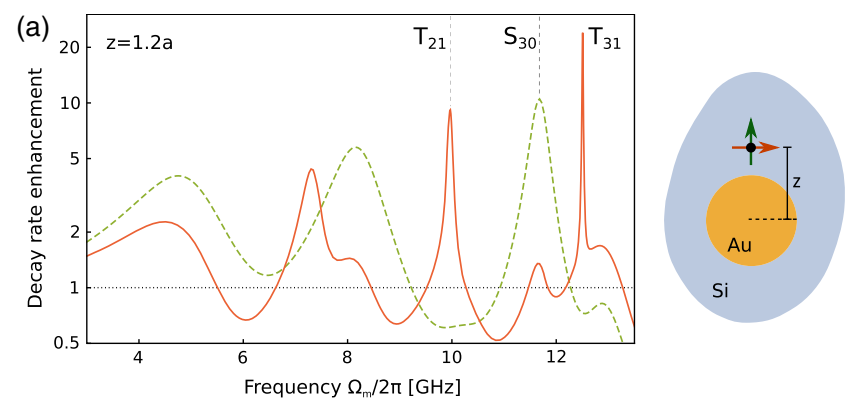

(b)

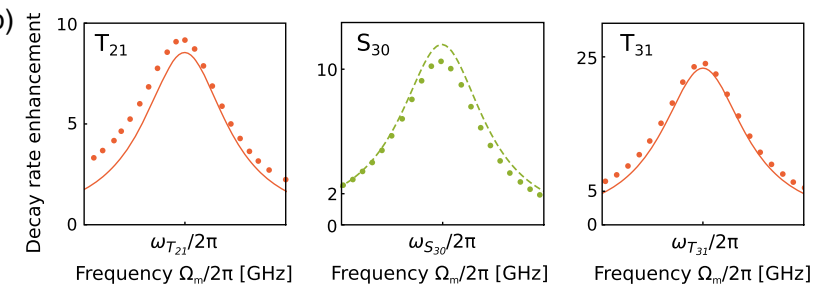

FIG. 3. Decay rate enhancement for an elastic emitter near a nanosphere. (a) Spectrum of decay rate enhancement $\Gamma / \Gamma_{0}[$ Eq. (7)] for the emitter oriented radially (green dashed line) or azimuthally (red solid line). (b) Magnified regions around three modes: $T_{21}, S_{30}$, and $T_{31}$ coupled to by an azimuthally $\left(T_{21}\right.$ and $\left.T_{31}\right)$ or radially $\left(S_{30}\right)$ polarized emitter. Points represent the exact calculations [Eq. (7)], while the solid lines describe coupling to the quasinormal modes of the resonator [Eq. (10)].

Consequently, the rate of energy dissipation $\gamma$ from the elastic emitter $\mathbf{f}$ near the antenna will be enhanced or quenched with respect to the free-space rate $\gamma_{0}$ following $\gamma / \gamma_{0}=P / P_{0}$. We note that alternate implementations of an elastic emitter necessarily call for a modification of the expression for the emitter-antenna interaction of Eq. (4) and the radiation power of Eq. (7). For example, spatially localized Brillouin scattering-induced lateral vibrations of a waveguide [5], or orbital transitions in $\mathrm{SiV}$ defects in diamonds, would need to be modeled as quadrupolar elastic sources coupled to a strain tensor [17] rather than displacement field. Nevertheless, (an appropriately modified) Eq. (7) can be used to extract the total LDES from the trace of the complete Green function $\operatorname{Tr}\left\{\operatorname{Im}\left[\mathbf{G}\left(\mathbf{r}_{m}, \mathbf{r}_{m}\right)\right]\right\}$ [34].

As an example, let us consider the decay rate enhancement $\gamma / \gamma_{0}$ of a dipolar emitter positioned $z=1.2 a$ from the center of the previously analyzed spherical gold scatterer embedded in silicon, shown schematically in Fig. 3(a). By tracing the spectral positions of the features in the spectrum corresponding to the emitter oriented radially, we can identify the enhancement and suppression of the emission as originating from the coupling with the low- $Q$ mixed QNMs $S_{n l}$ [26]. Spectra for the azimuthally oriented emitter indicate additional, significant enhancements due to the coupling with higher- $Q$ shear QNMs $\left(T_{n l}\right)$. These assertions regarding the nature of the excited QNMs originate solely from the identification of the peak frequencies since the underlying Eq. (7) does not readily provide insight into the modal structure of the enhancement spectra.

It is thus instructive to consider a modal picture of the coupling between a localized emitter and the QNMs of an elastic nanoresonator. Such a picture completes the analogy with the optical Purcell factor [21,22,37-39], originally introduced through the formalism of quantum electrodynamics, and enables consideration of nonclassical phonon emitters such as piezoelectric microantennas coupled to superconducting qubits [16], photonic crystals in the single-phonon regime [30], or $\mathrm{GHz}$ transitions in color centers in diamond [40]. To this end, we seek the elastic Purcell factor $F_{\alpha}$ which characterizes a single mode $\alpha$ of the cavity. As Koenderink [22] pointed out while analyzing plasmonic cavities, the Purcell factor provides a reasonable approximation of the rate enhancement only if (i) the normal modes of the system can be identified and (ii) one of these modes is dominant. Since we have already found the QNMs of the system, and recognized cases where the high- $Q$ well-separated shear modes dominate the response, we adopt this framework. However, since the Purcell factor is traditionally expressed solely in terms of the characteristics of a single cavity mode, assuming optimal positioning or orientation of the emitter, the Purcell factor should be treated as an upper bound for the spontaneous rate enhancement factor $\gamma / \gamma_{0}$. We address this limitation later.

We introduce the expansion of the nanoresonator displacement field into quantized QNMs [4]: $\hat{\mathbf{u}}(\mathbf{r})=$ $\sum_{\alpha} \hat{\mathbf{u}}_{\alpha}(\mathbf{r})=\sum_{\alpha} \sqrt{\hbar /\left(2 \Omega_{\alpha} \rho(\mathbf{r})\right)}\left[\hat{b}_{\alpha} \mathbf{U}_{\alpha}(\mathbf{r})+\right.$ H.c. $]$, where the displacement field is represented as a sum over modes $\alpha$ with resonant frequencies $\Omega_{\alpha}$, decay rates $\kappa_{\alpha}$, normalized mode profiles $\mathbf{U}_{\alpha}$, and phonon creation (annihilation) operators $\hat{b}_{\alpha}^{\dagger}\left(\hat{b}_{\alpha}\right)$. An interaction of the modes with the quantum emitter positioned at $\mathbf{r}_{m}$, described as a two-level system $\hat{\mathbf{f}}=\tilde{\mathbf{f}}(|g\rangle\langle e|+| e\rangle\langle g|)$, is expressed by a Hamiltonian $\hat{H}_{I}=\hat{\mathbf{f}} \cdot \hat{\mathbf{u}}\left(\mathbf{r}_{m}\right)$. Formally, the elastic Purcell factor is then defined as the ratio of the upper bound for the rate of spontaneous emission $\gamma_{\alpha}$ of the emitter excitation into the cavity mode $\alpha$ and $\gamma_{0}[38,41]$ :

$$
F_{\alpha}=\frac{\gamma_{\alpha}}{\gamma_{0}}=\frac{6 \pi}{\rho_{2} \rho_{1}^{1 / 2} \phi_{1}} \frac{Q_{\alpha}}{\Omega_{\alpha}^{3} V_{\alpha, \mathrm{eff}}},
$$

where $\phi_{i}=\mu_{i}^{-3 / 2}+\frac{1}{2}\left(2 \mu_{i}+\lambda_{i}\right)^{-3 / 2}$ can be interpreted as a screening factor, the quality factor of the resonator $Q_{\alpha}$ is given by $\Omega_{\alpha} / \kappa_{\alpha}$, and the mode displacement field defines the effective mode volume:

$$
V_{\mathrm{eff}, \alpha}=\frac{\int d \mathbf{r} \rho(\mathbf{r})\left|\mathbf{u}_{\alpha}(\mathbf{r})\right|^{2}}{\max \left[\rho(\mathbf{r})\left|\mathbf{u}_{\alpha}(\mathbf{r})\right|^{2}\right]}
$$


Interestingly, $F_{\alpha}$ has a form very similar to its electromagnetic counterpart, even exhibiting $\Omega_{\alpha}^{-3}$ dependence despite the marked dispersion differences of the free-space radiation power $P_{0}$ pointed out earlier. A detailed derivation of $F_{\alpha}$ and $V_{\text {eff, } \alpha}$ is given in Appendix D in the Supplemental Material [25]. We note that an equivalent expression for $V_{\text {eff, } \alpha}$ was derived by Eichenfield et al. [3] in an alternative way by analyzing the energy density in optomechanical crystals.

We can thus calculate the characteristics of a few lowest-order modes of gold scatterers in silicon, marked in Fig. 3(a) with dashed lines. For the pure shear $T_{21}$ mode centered at $\Omega_{T_{21}} / 2 \pi \approx 9.97 \mathrm{GHz}$, the effective mode volume $V_{\text {eff, } T_{21}} / V_{\text {geom }} \approx 0.25$ and the quality factor $Q_{T_{21}} \approx$ 90 yield the Purcell factor $F_{T_{21}} \approx 109$. Similarly, for the $T_{31}$ mode, the parameters are $\Omega_{T_{31}} / 2 \pi \approx 12.5 \mathrm{GHz}$, $V_{\text {eff }, T_{31}} / V_{\text {geom }} \approx 0.17, Q_{T_{31}} \approx 550$, and $F_{T_{31}} \approx 480$. Finally, for the mixed mode $S_{30}$, we find $\Omega_{S_{30}} / 2 \pi \approx 11.69 \mathrm{GHz}$, $V_{\text {eff }, S_{30}} / V_{\text {geom }} \approx 0.17, Q_{S_{30}} \approx 28$, and $F_{T_{30}} \approx 32$. These results indicate that the considerable Purcell factors stem, as expected for the low-order Mie resonances [13], from high $Q_{\alpha} \mathrm{s}$ of the QNMs rather than reduced effective mode volumes $V_{\text {eff }, \alpha}$.

We note that while neither silicon nor gold are isotropic materials [42] (characterized by elastic anisotropic ratios $\mathrm{A}_{\mathrm{Si}} \approx 1.6$ and $\mathrm{A}_{\mathrm{Au}} \approx 0.6$ ), their simplified description does not incur significant shifts of resonant frequencies nor changes in Purcell factors. Additionally, we have verified by full numerical calculations that when viscous damping in gold is included through the complex Young's modulus [43] the quality factors and the Purcell factors characterizing higher-order elastic modes are significantly quenched. While further studies of viscous damping are required to understand its exact magnitude, this mechanism will likely promote the use of lowest-order elastic modes in nanostructures for enhancing radiative emission from localized emitters.

As we have noted earlier, the Purcell factors are upper bounds for the decay rate enhancements, and so the estimated values of $F_{\alpha}$ are significantly larger than those calculated with Eq. (7) and shown in Fig. 3(a). This difference is mostly due to the fact that much like for dielectric high-refractive index electromagnetic Mie scatterers [13] the displacement field in the investigated cases is localized in the slow medium, i.e., gold, where the longitudinal and shear waves propagate $v_{l 1} / v_{l 2} \approx 2.7$ and $v_{s 1} / v_{s 2} \approx 4.3$ times slower than in the environment, respectively. Achieving such ratios of velocities between the medium and the antenna is difficult in electromagnetic systems.

To improve on the accuracy of this approach, it is instructive to modify the definition of the Purcell factor, introducing the dependence on the polarization, position, and the detuning of the emitter with respect to the cavity resonance $\Omega_{\alpha}$ (see Appendix D in the Supplemental Material [25]):

$$
\tilde{F}_{\alpha}\left(\mathbf{r}_{m}\right)=F_{\alpha} \frac{\Omega_{\alpha}}{\Omega_{m}} \frac{\left|\tilde{\mathbf{f}} \cdot \mathbf{u}_{\alpha}\left(\mathbf{r}_{m}\right)\right|^{2}}{|\tilde{\mathbf{f}}|^{2} \max _{\mathbf{r}}\left|\mathbf{u}_{\alpha}(\mathbf{r})\right|^{2}} \frac{\left(\kappa_{\alpha} / 2\right)^{2}}{\left(\Omega_{\alpha}-\Omega_{m}\right)^{2}+\left(\kappa_{\alpha} / 2\right)^{2}}
$$

Functions $\tilde{F}_{\alpha}\left(\mathbf{r}_{m}\right)$, calculated for the shear modes and mixed modes of the gold scatterer, are shown in the three panels of Fig. 3(b) with lines and correspond well to the exact results obtained from Eq. (7) depicted with points. The discrepancies found for the two lower $Q$ modes $T_{21}$ and $S_{30}$ are due to the additional coupling of the emitter with other QNMs and the continuum of free-space radiation modes.

In conclusion, we have introduced the formalism inherited from quantum electrodynamics to characterize elastic nanoresonators and found that such structures support modes with large Purcell factors determined by considerable $Q$ s rather than significantly reduced effective mode volumes. In particular, submicron gold particles, widely used in nanooptics, can readily enhance of spontaneous emission from phononic GHz emitters. Such elastic nanoantennas offer robust spatial and spectral control over the dissipation of mechanical vibrations and can be therefore used to introduce subwavelength control of phonon dynamics to various mechanical $[5,6,44]$ and optomechanical systems $[3,29]$.

We acknowledge support from the Australian Research Council (ARC) Discovery Project No. DP160101691 and ARC Centre for Ultrahigh Bandwidth Devices for Optical Systems (CUDOS) (Project No. CE110001018).

Note added in proof.-Recently related contributions were brought to our attention $[45,46]$.

*mikolaj.schmidt@mq.edu.au

[1] E. Purcell, Phys. Rev. 69, 674 (1946).

[2] L. Langguth, R. Fleury, A. Alù, and A. F. Koenderink, Phys. Rev. Lett. 116, 224301 (2016).

[3] M. Eichenfield, J. Chan, R. M. Camacho, K. J. Vahala, and O. Painter, Nature (London) 462, 78 (2009).

[4] J. E. Sipe and M. J. Steel, New J. Phys. 18, 045004 (2016).

[5] M. Merklein, B. Stiller, K. Vu, S. J. Madden, and B. J. Eggleton, Nat. Commun. 8, 574 (2017).

[6] R. O. Behunin, P. Kharel, W. H. Renninger, and P.T. Rakich, Nat. Mater. 16, 315 (2017).

[7] J. J. Faran Jr., J. Acoust. Soc. Am. 23, 405 (1951).

[8] C. Ying and R. Truell, J. Appl. Phys. 27, 1086 (1956).

[9] N. G. Einspruch, E. Witterholt, and R. Truell, J. Appl. Phys. 31, 806 (1960).

[10] Y.-H. Pao, C.-C. Mow, and J. Achenbach, J. Appl. Mech. 40, 872 (1973).

[11] J. Achenbach, Wave Propagation in Elastic Solids (Elsevier, New York, 2012), Vol. 16.

[12] K. F. Graff, Wave Motion in Elastic Solids (Courier Corporation, North Chelmsford, MA, 2012). 
[13] M. K. Schmidt, R. Esteban, J. J. Sáenz, I. Suárez-Lacalle, S. Mackowski, and J. Aizpurua, Opt. Express 20, 13636 (2012).

[14] B. A. Auld, Acoustic Fields and Waves in Solids (John Wiley \& Sons, New York, 1973).

[15] M. J. A. Schuetz, E. M. Kessler, G. Giedke, L. M. K. Vandersypen, M. D. Lukin, and J. I. Cirac, Phys. Rev. X 5, 031031 (2015).

[16] Y. Chu, P. Kharel, W. H. Renninger, L. D. Burkhart, L. Frunzio, P. T. Rakich, and R. J. Schoelkopf, Science 358, 199 (2017).

[17] R. Ruskov and C. Tahan, Phys. Rev. B 88, 064308 (2013).

[18] A. Ahmed, M. Pelton, and J. R. Guest, ACS Nano 11, 9360 (2017).

[19] K. Yu, P. Zijlstra, J. E. Sader, Q.-H. Xu, and M. Orrit, Nano Lett. 13, 2710 (2013).

[20] M. Pelton, Y. Wang, D. Gosztola, and J. E. Sader, J. Phys. Chem. C 115, 23732 (2011).

[21] C. Sauvan, J. P. Hugonin, I. S. Maksymov, and P. Lalanne, Phys. Rev. Lett. 110, 237401 (2013).

[22] A. F. Koenderink, Opt. Lett. 35, 4208 (2010).

[23] E. C. L. Ru and P. G. Etchegoin, Principles of SurfaceEnhanced Raman Spectroscopy (Elsevier, Amsterdam, 2009).

[24] C. F. Bohren and D. R. Huffman, Absorption and Scattering of Light by Small Particles (John Wiley \& Sons, New York, 2008).

[25] See Supplemental Material at http://link.aps.org/supplemental/ 10.1103/PhysRevLett.121.064301 for Appendixes.

[26] According to the conventional notation, the second subscript $l$ numbers the roots of the characteristic equation. For shear modes for $n \neq 0, l-1$ is replaced by $l$.

[27] M. J. Weber, Handbook of Optical Materials (CRC Press, Boca Raton, FL, 2002), Vol 19.

[28] M. Merklein, I. V. Kabakova, T. F. Büttner, D.-Y. Choi, B. Luther-Davies, S. J. Madden, and B. J. Eggleton, Nat. Commun. 6, 6396 (2015).

[29] K. Vahala, M. Herrmann, S. Knünz, V. Batteiger, G. Saathoff, T. Hänsch, and T. Udem, Nat. Phys. 5, 682 (2009).
[30] S. Hong, R. Riedinger, I. Marinković, A. Wallucks, S. G. Hofer, R. A. Norte, M. Aspelmeyer, and S. Gröblacher, Science 358, 203 (2017).

[31] S. Forstner, S. Prams, J. Knittel, E. D. Van Ooijen, J. D. Swaim, G. I. Harris, A. Szorkovszky, W. P. Bowen, and H. Rubinsztein-Dunlop, Phys. Rev. Lett. 108, 120801 (2012).

[32] V. Jain, J. Gieseler, C. Moritz, C. Dellago, R. Quidant, and L. Novotny, Phys. Rev. Lett. 116, 243601 (2016).

[33] M. Aspelmeyer, T. J. Kippenberg, and F. Marquardt, Rev. Mod. Phys. 86, 1391 (2014).

[34] L. Novotny and B. Hecht, Principles of Nano-Optics (Cambridge University Press, Cambridge, England, 2012).

[35] R. Snieder, in Scattering and Inverse Scattering in Pure and Applied Science, edited by R. Pike and P. Sabatier (Academic Press, San Diego, 2002), Chap. 1.7.1, p. 528.

[36] A. Ben-Menahem and S. J. Singh, Seismic Waves and Sources (Springer Science \& Business Media, New York, 2012).

[37] X. Zambrana-Puyalto and N. Bonod, Phys. Rev. B 91, 195422 (2015).

[38] R. Esteban, J. Aizpurua, and G. W. Bryant, New J. Phys. 16, 013052 (2014).

[39] H. Mertens, A. F. Koenderink, and A. Polman, Phys. Rev. B 76, 115123 (2007).

[40] K. V. Kepesidis, M.-A. Lemonde, A. Norambuena, J. R. Maze, and P. Rabl, Phys. Rev. B 94, 214115 (2016).

[41] A. Auffèves, D. Gerace, J.-M. Gérard, M. F. Santos, L. C. Andreani, and J.-P. Poizat, Phys. Rev. B 81, 245419 (2010).

[42] L. Saviot and D. B. Murray, Phys. Rev. B 79, 214101 (2009).

[43] C. Yi, M.-N. Su, P. D. Dongare, D. Chakraborty, Y.-Y. Cai, D. M. Marolf, R. N. Kress, B. Ostovar, L. J. Tauzin, F. Wen, W.-S. Chang, M. R. Jones, J. E. Sader, N. J. Halas, and S. Link, Nano Lett. 18, 3494 (2018).

[44] M. Maldovan, Nature (London) 503, 209 (2013).

[45] M. Landi, J. Zhao, W. E. Prather, Y. Wu, and L. Zhang, Phys. Rev. Lett. 120, 114301 (2018).

[46] J. Zhao, L. Zhang, and Y. Wu, J. Acoust. Soc. Am. 142, EL24 (2017). 